\title{
Guest Editorial Uniquely Human
}

\author{
Susan Bartos, PhD, RN, CCRN \\ Egan School of Nursing and Health Studies \\ Fairfield University \\ Fairfield, Connecticut 06824
}

Today, our world is not well. The World Health Organization (WHO) declared an official pandemic as COVID19 is spreading and affecting global populations. News outlets and social media have been taken over by epidemiological updates. Conversations are steered in a direction of fear, discontent, and even outrage. Casual exchanges between friends and acquaintances have been consumed with the need to understand what is happening. Simple small talk or the question of, "How are you doing," is no longer a short, polite answer. It is an open door for commiserating.

It is nearly impossible to not reflect on one's own health while COVID-19 dominates the headlines. There is a constant influx of new information and technologies to give us data on how to improve our own wellbeing. These are easily and readily available for download at the touch of a button - apps to measure caloric intake, to track sleeping patterns, to manage anxiety, or to visualize patterns in alcohol and tobacco behaviors. Now, at the mercy of a virus, we are being forced to face what we have been refusing to look towards: our own mortality.

There is not an app for that.

To understand health, we must first understand wellness, as their definitions set nestled within each other. According the Oxford English Dictionary, wellness is "the state of being in good health," while health is "the state of being free from illness or injury or a person's mental or physical condition." As healthcare providers, we coach others to craft goals that are "SMART" (Specific, Measurable, Attainable, Relevant, and Timely), yet how does one know when the goal of health and wellness has been achieved? How many goals can we set to improve our health and wellness? At what point do these goals surpass freedom from illness and injury and flirt with the field of immortality?

Staying in a state of self-defined good health through recommended and evidence-based behaviors is important, especially now. Self-care and wellness are often partnered together and self-care practices are endorsed by multiple organizations. Additionally, it is essential to find purpose, to create bonds, and to share in experiences to foster a state of human flourishing even in times of a global pandemic. The challenge is now to find connection through the quarantine, to not be afraid of our neighbor, but to find the power in loving them. To maintain a state of happiness and wellbeing because feeling good is feeling powerful - and feeling powerful is a superhuman feeling.

As our world zooms in around virulent outbreaks, the borders between healthy and well - sick and ill - become more defined. Often, health and wellness are placed on a linear continuum: We speak of ourselves, our patients, and our communities, as shifting from one side of the continuum to the other, from a state of poor health to a state of optimal health. But is it really that simple? Is health shaped in such a linear direction, from left to right or up and down - when we humans, are three, or perhaps even four-dimensional beings? We exist in spacetime, we live on a rock, being hurtled through a galaxy of other rocks. We are affected by gravity, by physics, by delicate and unpredictable biology, and by the same fundamental chemistry that started life as we know it. Our human cells contain 
the same atoms which are found deep in the cosmos of outer space. Yet, we simplify our wellbeing onto a black and white, two-dimensional string-like shape. Healthy or well. Sick or ill. Alive or dead.

Health and wellness are two words that are much more expansive than simple definitions. They are ways to recognize and embrace our limitations, to release perfectionistic ideas and tendencies, and to demonstrate the same level of empathy and understanding to ourselves that we as healthcare providers do for our global community. Grasping the notion of our start and of our end begins to form our new human shape. What shapes us is what makes us weak, strong, hungry, scared, joyful, or indifferent. It makes us: us.

COVID-19 is a challenge to us because it is threatening the clean and flawless shape of our health continuum. It is exposing vulnerabilities in our strongest, in our systems, and in our selves. Our shape is being exposed and what we are being left with, is the shape of what is to come, and it is much different than before. It is disorganized, it is more chaotic, but the patterns of our lifestyle and of our delicate humanity still exist within that chaos. This shape is multidimensional. It is made of human flourishing, how we thrive, and how we appreciate the ever-changing process. This shape is not linear. It is not circular - it may not even be a direction or shape of which you or I are ready to recognize or define - but it is our shape and what will always remain is what makes us uniquely human.

Address author correspondence to:

Susan Bartos, PhD, RN, CCRN

Egan School of Nursing and Health Studies

Fairfield University

1073 North Benson Road

Fairfield, Connecticut 06824

sbartos@fairfield.edu 